\title{
Relevamiento de Competencias Clínicas Básicas para la Psicoterapia según expertos argentinos
}

\author{
Lia Nadia Crocamo ${ }^{1}$ y Denise Benatuil
}

\begin{abstract}
RESUMEN
En las últimas décadas, han aumentado los estudios sobre las competencias para el ejercicio de profesiones de la salud (Gonsalvez et al., 2017). En psicología y, en particular para la psicoterapia, se han elaborado diferentes escritos que detallan las competencias que deben adquirir los psicoterapeutas durante su formación. Sin embargo, a nivel local no se cuenta con estudios sistemáticos específicos. Con el objetivo de conocer cuáles son las competencias más relevantes que deben poseer los psicoterapeutas argentinos para el ejercicio de la psicoterapia, se realizaron 20 entrevistas semi-dirigidas sometidas a un análisis temático. Se encontraron siete competencias clínicas básicas que fueron denominadas Proceso diagnóstico, Diseño, intervenciones y técnicas, Evaluación y finalización del proceso, Vínculo terapéutico, Rol profesional, Variables contextuales y diversidad y Manejo de entrevista.
\end{abstract}

Palabras clave: competencias clínicas, psicoterapia, entrevistas semi-estructuradas, análisis temático.

\section{Survey of Basic Clinical Competences for Psychotherapy according to Argentine experts}

\begin{abstract}
In recent decades, studies on the competences for the exercise of health professions have increased (Gonsalvez, Hamid, Savage, \& Livni, 2017). In psychology and, in particular, for psychotherapy, different writings have been prepared detailing the competences that psychotherapists must acquire during their training. However, at the local level there are no specific systematic studies. With the objective of knowing which of the most relevant competences that Argentine psychotherapists must possess for the exercise of psychotherapy, 20 semi-directed interviews were carried out, subject to a thematic analysis. Seven basic clinical competences were found that were called Diagnostic process, Design, interventions and techniques, Evaluation and completion of the process, Therapeutic link, Professional role, Contextual variables and diversity, and Interview management.
\end{abstract}

Keywords: clinical skills, psychotherapy, semi-structured interview, thematic analysis.

\footnotetext{
1 Instituto Nacional de Tecnología Industrial, Argentina; liacrocamo@gmail.com; http://orcid.org/00000002-2199-8709

2 Universidad de Buenos Aires, Argentina; denisebenatuil66@gmail.com; http://orcid.org/0000-0001-82513982
} 
Numerosos autores se han acercado al estudio de las competencias y, en línea con ello, existen múltiples definiciones de acuerdo con el ámbito de aplicación (Martínez \& Carmona, 2009). En la Declaración Internacional de Competencias Fundamentales en Psicologia Profesional (International Association of Applied Psychology, 2016) se afirma que se trata de agrupaciones de conocimientos, habilidades, capacidades y otros atributos que le permiten a una persona lograr actuar de manera eficaz y dentro de un estándar definido, en una situación particular de su ejercicio profesional.

A partir de los años 70, comienzan una serie de debates acerca de la formación de los psicólogos desde una perspectiva basada en competencias, lo que permitió contar con un detalle más claro de aquellos conocimientos, habilidades, actitudes y valores que los psicoterapeutas deberian desarrollar (Kenkel \& Peterson, 2010), y en especial, en las dos últimas décadas, han aumentado los estudios sobre las competencias para el ejercicio de profesiones de la salud (Gonsalvez, Hamid, Savage, \& Livni 2017). En psicología y, en particular para la psicoterapia, se han elaborado diferentes modelos que detallan las competencias que deben adquirir los psicoterapeutas durante su formación.

En Estados Unidos, la American Psychological Association elaboró el Modelo del Cubo (Daniel, Roysircar, Abeles, \& Boyd, 2004; Rodolfa et al., 2005), en Europa, el modelo de formación de la Unión Europea, EuroPsy que otorga el European Diploma in Psychology (European Certificate in Psychology, 2011) y la European Association for Psychotherapy (EAP) elaboró un modelo detallando competencias primarias (European Association for Psychotherapy, 2017), y, en América Latina, el Proyecto Tuning (Beneitone et al., 2007; ver tabla 1). En otros países como Canadá, la Canadian Psychological Association (CPA) propuso un modelo de seis competencias nucleares (Mutual Recognition Agreement, 2004). En Australia, la Australian Psychological Society (APS) elaboró un listado de atributos que los estudiantes deben desarrollar en los primeros años en la universidad y que sirven como referencia para la acreditación del psicólogo australiano (Cranney et al., 2009). En Nueva Zelandia, la New Zealand Psychologists Board (NZPB) realizó una guía de competencias nucleares que representan los requisitos mínimos para la práctica de la psicología (New Zealand Psychologists Board, 2011). Por último, en España, la Agencia Nacional de Evaluación de la Calidad y Acreditación de España, (ANECA) elaboró una guía de reflexión acerca de la formación de psicólogos, el Libro Blanco de la Carrera de Psicologia (ANECA, 2005; ver tabla 2).

Tabla 1.

Modelos de competencias internacionales. 


\begin{tabular}{|c|c|}
\hline \multirow{2}{*}{ Modelo del Cubo } & $\begin{array}{l}\text { Competencias fundamentales } \\
\text { a. Práctica reflexiva } \\
\text { b. Métodos y conocimiento científico } \\
\text { c. Relaciones } \\
\text { d. Estándares éticos y legales } \\
\text { e. Diversidad individual y cultural } \\
\text { f. Sistemas interdisciplinarios }\end{array}$ \\
\hline & $\begin{array}{l}\text { Competencias funcionales } \\
\text { a. Diagnóstico, evaluación y conceptualización del caso } \\
\text { b. Intervención } \\
\text { c. Consultoría } \\
\text { d. Investigación } \\
\text { e. Supervisión y enseñanza } \\
\text { f. Administración (Daniel et al., 2004; Rodolfa et al., 2005) }\end{array}$ \\
\hline EuroPsy & $\begin{array}{l}\text { Competencias primarias } \\
\text { a. Especificación de objetivos } \\
\text { b. Evaluación } \\
\text { c. Desarrollo } \\
\text { d. Intervención } \\
\text { e. Valoración } \\
\text { f. Comunicación } \\
\text { (European Certificate in Psychology, 2011) }\end{array}$ \\
\hline $\begin{array}{l}\text { European Association for } \\
\text { Psychotherapy }\end{array}$ & $\begin{array}{l}\text { Dominios de competencias nucleares } \\
\text { a. Práctica profesional autónoma y responsable } \\
\text { b. Relación terapéutica } \\
\text { c. Evaluación, diagnóstico y conceptualización } \\
\text { d. Desarrollo de metas, planes y estrategias } \\
\text { e. Técnicas e intervenciones } \\
\text { f. Manejo del cambio, crisis y trauma } \\
\text { g. Evaluación y finalización de la psicoterapia } \\
\text { h. Colaboración con otros profesionales } \\
\text { i. Supervisión } \\
\text { j. Ética y cultura } \\
\text { k. Gestión y administración } \\
\text { l. Investigación } \\
\text { m. Prevención y educación (European Association for Psychotherapy, } \\
\text { 2017) }\end{array}$ \\
\hline Proyecto Tuning & $\begin{array}{l}27 \text { competencias, entre ellas: } \\
\text { a. Realización de diagnósticos y evaluaciones psicológicas } \\
\text { b. Comprensión e intervención ante los problemas psicológicos de los } \\
\text { seres humanos de acuerdo con su contexto histórico, social, cultural y } \\
\text { económico } \\
\text { c. Diseño, ejecución y evaluación de técnicas y estrategias de } \\
\text { intervención en diferentes campos de acción de la Psicología } \\
\text { (Beneitone et al., 2007) }\end{array}$ \\
\hline
\end{tabular}

Tabla 2.

Otros modelos de competencias. 


\begin{tabular}{|c|c|}
\hline $\begin{array}{l}\text { Canadian Psychological } \\
\text { Association }\end{array}$ & $\begin{array}{l}\text { Competencias nucleares } \\
\text { a. Relaciones interpersonales } \\
\text { b. Evaluación y diagnóstico } \\
\text { c. Intervención y Orientación } \\
\text { d. Investigación } \\
\text { e. Estándares éticos } \\
\text { f. Supervisión (Mutual Recognition Agreement, 2004) }\end{array}$ \\
\hline $\begin{array}{l}\text { Australian Psychological } \\
\text { Society }\end{array}$ & $\begin{array}{l}\text { Atributos } \\
\text { a. Conocimiento y comprensión } \\
\text { b. Métodos de investigación } \\
\text { c. Habilidades de pensamiento crítico } \\
\text { d. Valores } \\
\text { e. Habilidades de comunicación } \\
\text { f. Aprendizaje y aplicación (Cranney et al., 2009) }\end{array}$ \\
\hline $\begin{array}{l}\text { New Zealand Psychologists } \\
\text { Board }\end{array}$ & $\begin{array}{l}\text { Competencias nucleares } \\
\text { a. Disciplina, conocimiento e investigación } \\
\text { b. Práctica profesional, legal y ética } \\
\text { c. Encuadre, medición y planificación } \\
\text { d. Intervención y aplicación de un modelo terapéutico } \\
\text { e. Comunicación } \\
\text { f. Relaciones con la comunidad y profesionales } \\
\text { g. Orientación y colaboración } \\
\text { h. Práctica reflexiva, } \\
\text { i. Supervisión } \\
\text { j. Diversidad, cultura y tratado de Waitangi (New Zealand Psychologists } \\
\text { Board, 2011) }\end{array}$ \\
\hline $\begin{array}{l}\text { Agencia Nacional de } \\
\text { Evaluación de la Calidad y } \\
\text { Acreditación de España }\end{array}$ & $\begin{array}{l}\text { Algunas competencias: } \\
\text { a. Conocer las funciones, características y limitaciones de los distintos } \\
\text { modelos teóricos de la psicología } \\
\text { b. Conocer distintos métodos de evaluación, diagnóstico y tratamientos } \\
\text { psicológicos en diferentes ámbitos aplicados de la Psicología (ANECA, } \\
2005 \text { ) }\end{array}$ \\
\hline
\end{tabular}

\section{Situación local}

Argentina es uno de los países que posee mayor número de psicólogos per cápita a nivel mundial. Se estima que durante el periodo 2017-2018 Argentina contaba con 82.776 psicólogos activos, $84 \%$ de sexo femenino frente a $16 \%$ masculino. La mayoría de los psicólogos se desempeñan en el área clínica (Klinar, Gago, \& Alonso, 2019). A pesar de esto, el accionar del psicólogo en el área clínica se reconoce por primera vez en el año 1985, mediante la Ley 23.277 del Ejercicio Profesional de la Psicologia, y por medio de la Resolución $\mathrm{N}^{\circ} 2447$ del Ministerio de Educación (Ministerio de Educación y Justicia, 1985). Previo a esta regulación, atento a la resolución $\mathrm{N}^{\circ} 2282 / 54$ del Ministerio de Salud Pública de la Nación, y la ley 17.132, se prohibía explícitamente el ejercicio de la psicoterapia por parte del psicólogo (Caló, 2000; González \& Dagfal, 2012). A partir de la Resolución Ministerial 136/04, la carrera de psicología fue declarada de interés público, puesto que su ejercicio compromete de modo directo la salud, la seguridad, los derechos, los bienes o la formación de los habitantes (Fierro \& Di Doménico, 2016). Por consiguiente, los planes de estudio y contenidos curriculares son evaluados periódicamente a través de la Comisión Nacional de Evaluación y Acreditación Universitaria (CONEAU) encargada de acreditar las carreras universitarias (Klappenbach, 2015).

En lo que respecta al estudio de competencias, la cantidad de estudios empíricos realizados ha sido escasa. En uno de ellos, se ha investigado la 
percepción de los estudiantes de psicología en relación con las competencias planteadas en el Proyecto Tuning América Latina y concluye que todas las competencias fueron consideradas importantes en la formación del psicólogo, a pesar de pertenecer a diferentes áreas de conocimiento. Además, se ha destacado la importancia del desarrollo ético de la profesión (Benatuil \& Laurito, 2017). Otra investigación cuyo objetivo fue analizar la distancia entre los niveles de importancia y logro autopercibido de las competencias específicas planteadas en el marco del Proyecto Tuning América Latina, encontró que en todas las competencias fue más alta la importancia que el nivel de logro alcanzado (Laurito \& Benatuil, 2017).

Sin embargo, en lo que respecta a la psicoterapia, no se cuenta con descripciones locales específicas, donde se detallen cuáles son las habilidades, actitudes, conductas y conocimientos que los profesionales deben adquirir durante la formación. Por lo tanto, como se hace necesario contar con mayor investigación local sobre el perfil que se requiere para el ejercicio de la psicoterapia, este estudio tiene como objetivo identificar las competencias clínicas básicas que deben poseer los psicoterapeutas para el ejercicio de la psicoterapia en Argentina.

\section{MÉTODO}

\section{Diseño y tipo de estudio}

Se utilizó la metodología cualitativa mediante un diseño no experimental, transversal de tipo exploratorio.

\section{Participantes}

La muestra estuvo conformada por 20 psicoterapeutas argentinos expertos, 8 mujeres y 12 hombres. Los criterios que debian cumplir para considerarse expertos eran: 1. Poseer como mínimo 30 años de dedicación continua al ejercicio clínico, 2. Poseer experiencia como formador de terapeutas en instituciones, 3. Estar a cargo de cátedras de material vinculadas al ejercicio de la psicoterapia en universidades públicas o privadas, 4. Desempeñarse como supervisor de otros terapeutas, 5. Desempeñarse en un cargo jerárquico de gestión de alguna institución que brinda asistencia psicoterapéutica, 6. Contar con reconocimiento nacional o internacional.

La media de experiencia de este grupo de profesionales era de 40 años, $D E=8.46$. Para obtener mayor grado de representatividad y heterogeneidad de respuestas, participaron referentes de diferentes modelos teóricos tradicionales: sistémico, humanístico-existencial, psicoanalitico, cognitivoconductual e integrativo (Fernández-Álvarez, 1994).

La cantidad de informantes clave estuvo determinada por el criterio de saturación teórica (Ardila Suárez \& Rueda Arenas, 2013; Corbin \& Strauss, 2002). Se observó que luego de entrevistar a 4 informantes por modelo teórico no surgian nuevas variaciones entre los datos, los nuevos temas codificados pertenecian a las mismas categorias ya encontradas.

Luego, se convocó a tres psicoterapeutas, con formación académica de nivel magister o superior- dos dedicados a la investigación en psicoterapia y uno a la formación por competencias de psicólogos-, para realizar un juicio de experto sobre el análisis temático de las entrevistas. 


\section{Instrumentos}

Se realizaron entrevistas semi-dirigidas individuales en profundidad, con una duración aproximada de 45 minutos, cuya finalidad era explorar las competencias clínicas básicas requeridas para el ejercicio de la psicoterapia. Los encuentros se organizaban en torno la pregunta ¿Cuáles son las competencias clínicas básicas que deberian poseer los psicoterapeutas para ejercer su profesión? Durante el encuentro, se solicitaba que mencionaran, explicaran y ejemplificaran las competencias, que enumerasen estos conocimientos, habilidades, actitudes y conductas, y que consideraran aquellos terapeutas con problemas de desempeño y las competencias que ellos no habrian adquirido.

\section{Análisis de datos}

Se realizó el proceso de análisis temático con rigor científico propuesto por Boyatzis (1998), siguiendo las etapas formuladas por Braun y Clarke (2006). Complementariamente, se calcularon porcentajes de acuerdo interjueces para el estudio de validez de contenido y de criterio de los resultados obtenidos (Herrera Rojas, 1993).

\section{Procedimiento}

Inicialmente, con la intención de identificar las competencias clínicas básicas que deben poseer los psicoterapeutas para el ejercicio de la profesión, se elaboró un listado de posibles terapeutas expertos para ser entrevistados. Con el objetivo de lograr mayor grado de representatividad de los enfoques clínicos vigentes en nuestro medio, se incluyeron profesionales de diferentes modelos teóricos tradicionales (Fernández-Álvarez, 1994). En primer lugar, los informantes clave fueron contactados por teléfono y/o correo electrónico. Una vez que se explicaba el propósito de la investigación, se aclaraba que la participación era anónima y voluntaria y se dejaba constancia de la conformidad para participar, se programaban las entrevistas.

Los encuentros con los informantes clave tenían una duración aproximada de 45 minutos y se llevaron a cabo en sus consultorios o instituciones de pertenencia. Se transmitía nuevamente a los participantes la finalidad del proyecto de investigación, los ejes que se explorarian, y se acordaba que la modalidad de registro de la información sería la grabación de audio.

Finalizado el encuentro, se realizaba la desgrabación de los audios de las entrevistas y se analizaba el material empleando el proceso de análisis temático y las fases propuestas por Braun y Clarke (2006). En simultáneo, para contribuir a la validez de contenido durante el proceso, se convocó a expertos en metodologia, para que realizaran un análisis temático a ciegas de tres entrevistas efectuadas a los informantes clave. Se enviaba por mail las desgrabaciones textuales y, finalizada la tarea, reenviaban el material por el mismo medio. Como resultado del análisis temático, realizado en conjunto con expertos, se establecieron las competencias clínicas básicas requeridas para el ejercicio profesional $y$ se realizaron las definiciones conceptuales y operacionales de cada una.

Cabe destacar que el estudio cumple todos los estándares éticos de la American Psychological Association (2010). Se informó el propósito de la investigación, la duración estimada y los procedimientos; su derecho a rehusarse a participar y retirarse de la investigación una vez que su 
participación haya comenzado; de la confidencialidad, precauciones razonables para proteger la información y, en lo que respecta a las grabaciones, se obtuvo el permiso de los participantes antes de grabar.

\section{RESULTADOS}

\section{Análisis temático de las entrevistas}

Con el objetivo de procesar la información obtenida durante los encuentros con expertos, y luego de realizar la transcripción textual de cada audio, se empleó el proceso de análisis temático con rigor científico propuesto por Boyatzis (1998). Para ello, se siguieron las fases propuestas por Braun y Clarke (2006) denominadas familiarización con los datos, generación de códigos iniciales, búsqueda y revisión de temas, definición y denominación de temas y producción del informe final.

\section{Familiarización con los datos y generación de códigos iniciales}

En primera instancia, una vez que se realizó la desgrabación de las entrevistas a los terapeutas expertos, se efectuó una lectura detenida y reiterada de la información con el fin de familiarizarse con el material. En simultáneo, se realizaron anotaciones de ideas generales, organizando la información en grupos de un mismo significado, denominados códigos iniciales (Braun \& Clarke, 2006).

\section{Búsqueda y revisión de temas}

En segunda instancia, se procedió a la búsqueda y revisión de temas. A partir de la definición de Boyatzis (1998), quien afirma que un tema es aquel que captura información importante en relación con las preguntas de investigación, representando un nivel de respuesta estructurada o significado. Tomando como base los códigos iniciales, se realizó un análisis exhaustivo de cada entrevista, relevando palabras, oraciones y párrafos que referian a competencias clínicas básicas para el ejercicio profesional.

Finalmente, se organizó la información en grupos de temas con un mismo significado, detallando los segmentos básicos de información en crudo que pudieran considerarse significativos con relación al tema bajo estudio. Los códigos iniciales se reorganizaron en torno a ocho temas principales (Boyatzis, 1998; Braun \& Clarke, 2006; ver tabla 3).

Tabla 3.

Búsqueda y revisión de temas.

\begin{tabular}{ll}
\hline Códigos iniciales & Grupos de temas \\
\hline Evaluación inicial & Proceso diagnóstico
\end{tabular}


Proceso terapéutico

Evaluación del proceso

Habilidades interpersonales

Características del terapeuta

Formación
Diseño, intervenciones y técnicas

Evaluación y finalización del proceso

Vínculo terapéutico

Manejo de entrevista

Rol profesional

Variables contextuales y diversidad

Formación de base y actualización

\section{Definición y denominación de temas}

Una vez que los códigos iniciales se reorganizaron en grupos de temas, se evaluó si cumplían con una serie de premisas para ser considerados competencias clínicas básicas para el ejercicio de la psicoterapia: 1. Que se tratara de conocimientos, habilidades, actitudes y conductas que remitieran al ejercicio profesional, 2. Que aparecieran como minimo en la mitad de las entrevistas a expertos, 3. Que fueran características generales para el ejercicio y que no se remitieran exclusivamente al trabajo con alguna población específica o modalidad terapéutica.

\section{Análisis temático por jueces expertos}

Tal como afirman Mieles Barrera, Tonon y Alvarado Salgado (2002), un análisis temático consistente requiere que al menos dos investigadores trabajen con la misma información, codificando cada uno por su lado -doble código- y que luego se comparen sus códigos entre sí. Para lograr este cometido, se convocó a tres jueces expertos en metodología, para que analizaran tres de las entrevistas más completas que se obtuvieron, es decir, aquellas de las que se extrajo la mayor cantidad de grupos de temas.

Una vez que acordaban participar, de manera anónima y voluntaria, se enviaba por mail la desgrabación textual de tres entrevistas.

En primer lugar, paso previo a la elaboración de la definición conceptual y operacional de cada variable, se compararon los resultados del análisis temático entre los jueces. Para ello, se elaboró un cuadro de doble entrada con la nominación de cada competencia, donde se ingresaba la información de los participantes. Como resultado, el proceso permitió no solo revisar el contenido previamente determinado, sino también incluir información adicional que no habia sido considerada de antemano. Ver tabla 4.

Tabla 4

Análisis temáticos por jueces expertos.

\begin{tabular}{lllll}
\hline $\begin{array}{l}\text { Competencia } \\
\text { clínica }\end{array}$ & Extracto entrevista 1 & Juez 1 & Juez 2 & Juez 3 \\
\hline
\end{tabular}




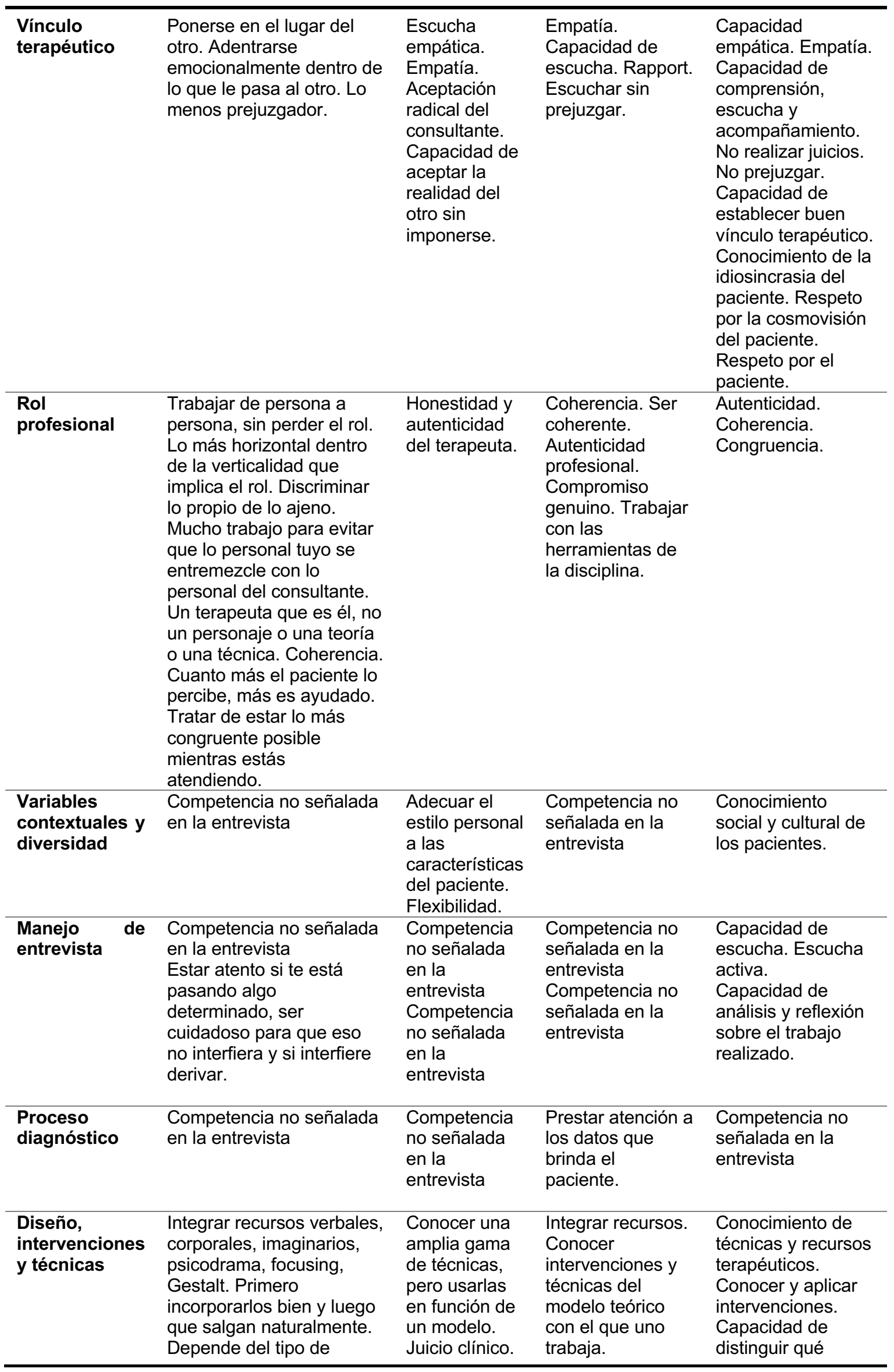


Experiencia técnica. Diseñar en función de lo que el paciente trae como datos. recursos e intervenciones son más adecuados para el paciente. Saber utilizar recursos terapéuticos. Capacidad de intervención. Ojo clínico.

\section{Producción del informe final}

Como resultado del proceso del análisis temático de las entrevistas con la participación de expertos en metodología, se identificaron siete competencias clínicas básicas requeridas para el ejercicio de la psicoterapia, cuyas definiciones conceptuales se exponen a continuación:

Proceso diagnóstico. Conocimientos, habilidades y técnicas requeridas para evaluar las dimensiones cognitivas, afectivas y conductuales del consultante, proceso que requeriría elaborar un diagnóstico, conceptualizar el caso, establecer el motivo de consulta, foco y objetivos terapéuticos, además de la formulación de hipótesis.

Diseño, intervenciones y técnicas. Elaboración del plan de tratamiento, selección y empleo de procedimientos para abordar diferentes condiciones clínicas, que involucra no solo el diseño de tratamiento, la selección y aplicación de técnicas e intervenciones fundamentadas, sino también la integración de los recursos disponibles.

Evaluación y finalización del proceso. Proceso de reflexión acerca de la propia actuación, procesos, intervenciones y resultados de la psicoterapia, que implicaría evaluar la eficacia tanto del proceso psicoterapéutico en su conjunto como de la persona del terapeuta, la consideración de sus limitaciones y la eventual necesidad de derivación.

Vinculo terapéutico. Establecimiento y sostén de las relaciones interpersonales con el consultante y sus allegados con el objetivo de concretar las metas de la psicoterapia.

Rol profesional. Habilitaciones e inhibiciones del rol, actitud de la persona del terapeuta frente a la tarea, regulación de la distancia interpersonal y dominio de los límites de la relación terapéutica, que implica la diferenciación de roles, la consistencia y coherencia en su actuar, y la consideración de los límites su labor y habilitación profesional.

Manejo de entrevista. Dominio de la técnica de comunicación interpersonal oral, iniciada con el propósito específico de obtener información, para tomar decisiones e identificar y clarificar la demanda en el marco de encuentros planificados, cuyo contenido incluye la recolección y análisis de información y la comunicación no verbal.

Variables contextuales y diversidad. Apertura a la diversidad étnica, cultural, religiosa, política, social, económica y familiar, que se refleja en la consideración de todos estos factores durante el desarrollo de todas las fases del proceso terapéutico.

Seguidamente, a partir de los extractos obtenidos en el análisis temático de las entrevistas, se elaboraron las definiciones operacionales que luego serían revisadas por expertos, tal como se detalla en el próximo apartado. 


\section{Revisión final por jueces expertos}

Por último, se realizó un juicio de expertos sobre el contenido de las entrevistas con el propósito de realizar ajustes en las definiciones conceptuales y operacionales de cada variable, si fuera necesario. Cabe señalar que el establecimiento de los acuerdos interjueces es uno de los componentes esenciales en la demostración de la calidad de las investigaciones, cuya ausencia generaria interpretaciones, conclusiones y resultados de menor grado de validez (Pedrosa, Suárez-Álvarez y García-Cueto, 2013; Urrutia Egaña, et al., 2014).

Con este cometido, se otorgaba a los jueces tres entrevistas desgrabadas, de las cuales se obtuvo una mayor cantidad de grupo de temas, y un listado de las siete competencias clínicas básicas para la psicoterapia, junto con sus definiciones conceptuales y operacionales. Se brindaba la consigna "A continuación se presenta un listado de competencias requeridas para el ejercicio de la psicoterapia, realice una lectura detenida de cada entrevista y señale la presencia (1) o ausencia (0) de cada concepto".

Luego, se calculaba el grado de acuerdo entre las respuestas de los participantes, se conservaria el contenido de las entrevistas cuyo consenso superara el 60\% tomando el criterio referido por Herrera Rojas (1993), y se reexaminaría lo de menor conformidad. Para su cálculo, se incluyeron las respuestas del autor de la investigación.

Como resultado, el porcentaje de acuerdo total de cada entrevista analizada fue superior al $75 \%$, motivo por el cual se conservaron las competencias previamente establecidas. Por otro lado, se excluyeron algunos fragmentos utilizados para la elaboración de las definiciones operacionales de Variables contextuales y diversidad y Evaluación y finalización del proceso, puesto que el grado de acuerdo de ese material fue menor al aceptable.

En síntesis, para la identificación de las competencias clínicas básicas que deben poseer los psicoterapeutas para el ejercicio de su profesión, se realizaron 20 entrevistas semi-dirigidas, que luego se sometieron a un análisis temático en el que, complementariamente, participaron expertos en metodologia, quienes actuaron como jueces en diferentes etapas del proceso. A partir de lo expuesto, se identificaron siete competencias denominadas Proceso diagnóstico, Diseño, intervenciones y técnicas, Vínculo terapéutico, Rol profesional, Variables contextuales y diversidad, Manejo de entrevista, Evaluación y finalización del proceso.

\section{COMENTARIOS}

En lineas generales, los resultados de esta investigación son similares a los encontrados en estudios realizados en otros contextos. Competencias tales como Proceso diagnóstico, Diseño intervenciones técnicas, Rol profesional, Variables contextuales y diversidad se encuentran presentes en la mayoría de los modelos internacionales (Daniel et al., 2004; Edwards, 2000; European Association for Psychotherapy, 2017; New Zealand Psychologists Board, 2011; Rodolfa et al., 2005). En ambos casos, las competencias clínicas descriptas coinciden con las fases del proceso psicoterapéutico, desde la primera consulta hasta la finalización del tratamiento (Fuentes Pallas \& Lorenzo Pontevedra, 2008).

Sin embargo, se observan algunas discrepancias entre los resultados obtenidos en esta investigación y estudios previos. Se observa que la población 
local enfatiza algunos elementos frente a otros, de modo que algunos componentes de competencias presentadas en otros modelos se constituyen como competencias por sí mismas en este estudio. Por último, también se observan diferencias que corresponderian a expresiones lingüísticas particulares de cada nación.

La competencia proceso de diagnóstico y el establecimiento de objetivos suelen ser conceptualizadas por separado en otros modelos (European Association for Psychotherapy, 2017; European Certificate in Psychology, 2011), pero en este estudio se concluye que formarian una unidad indivisible, es decir, los conocimientos, habilidades, actitudes y conductas requeridas para la evaluación de las dimensiones del consultante, la conceptualización del caso, y el establecimiento de objetivos de tratamiento -si es que se requiere iniciarlo- son parte de un mismo proceso. Al igual que lo detallado en estudios preexistentes, la competencia Diseño, intervenciones y técnicas se operacionaliza mediante la toma de decisiones acerca del tipo de tratamiento, formato, duración estimada, objetivos y expectativas de resultado (FernándezÁlvarez, Pérez, \& Fraga Míguez, 2008; García \& Fernández-Álvarez, 2001). En los modelos internacionales, se observa que suelen conceptualizarse como competencias diferentes, por un lado, la planificación del proceso psicoterapéutico, y por otro, la utilización de las técnicas e intervenciones. Se desprende de los resultados obtenidos en este estudio que el plan de tratamiento elaborado está indefectiblemente relacionado de manera directa con las técnicas que se seleccionarán para lograr los objetivos planteados a partir del proceso diagnóstico realizado previamente.

Si bien Vínculo terapéutico y Evaluación y finalización del proceso están presentes implícitamente en algunos modelos internacionales, a partir del análisis de las entrevistas a terapeutas expertos locales, se observa que estas tareas revisten gran relevancia y que ameritan ser consideradas como una competencia en sí misma. Coincidente con numerosos estudios anteriores, los expertos entrevistados señalan la relación significativa entre la calidad de la alianza terapéutica y el desarrollo y resultados de la psicoterapia y la empatía como elemento esencial (Laws, et al., 2017; Zilcha-Mano, 2017).

Al igual que en otros modelos internacionales, en este estudio se encuentra que para el ejercicio de la psicoterapia se requiere la evaluación reflexiva sobre la propia práctica y el respeto de las incumbencias profesionales, en este estudio denominada competencia Rol profesional. La importancia otorgada a esta competencia puede explicarse por lo sucedido durante el año 2018. Mediante la resolución 1254/2018 publicada en el Boletín Oficial de la Nación, se modificaron resoluciones previas referidas a la incumbencia de diferentes carreras. Una de las reacciones más fuertes se produjo en las carreras de psicología, que pasó de contar de dieciséis a cinco actividades reservadas para el título. Por un lado, esta decisión generó repudio en diferentes Colegios de Psicólogos, al considerar que a partir de la nueva resolución se reducen las actividades profesionales que podrian ejercer los graduados (Colegio de Psicólogos de Tucumán, 2018). Mientras que otras instituciones comunicaron oficialmente que no se modificaria el desarrollo y ejercicio de la práctica profesional de la Psicología (Universidad Nacional de la Plata, 2018).

Por otra parte, y a diferencia de lo presentado en los modelos internacionales, se ha encontrado que en la población local la competencia Manejo de entrevista es una competencia por sí misma, mientras que en otros 
modelos como en el Modelo del Cubo, se conceptualiza dentro de la competencia fundamental Métodos y conocimiento científico (Daniel et al., 2004).

Del mismo modo, existe un acuerdo generalizado en cuanto todas las psicoterapias comparten factores comunes que explicarian el cambio en psicoterapia. Tanto en esta investigación como en los modelos internacionales se ha señalado la importancia del establecimiento de la alianza terapéutica (Botella \& Maestra, 2016).

En sintesis, los resultados obtenidos en esta investigación son similares a los plasmados por otros modelos de competencias internacionales (Bartram et al., 2008; Beneitone et al., 2007; Berdullas et al., 2007; European Association for Psychotherapy, 2017; Kaslow et al., 2007). En los modelos internacionales se hace hincapié fundamentalmente en la eficacia del proceso terapéutico y en la alineación entre la estrategia de tratamiento, las técnicas empleadas y los resultados obtenidos. En la población argentina, el auto monitoreo del terapeuta, la evaluación de su desempeño, el registro de sus limitaciones personales y la posibilidad de derivación cobra una importancia mayor, que no es mencionada en otros modelos. Probablemente, esta particularidad se deba a la tradición psicoanalitica de la psicoterapia en Argentina, modelo que históricamente ha enfatizado que el terapeuta debe tener un conocimiento profundo de sí mismo y ser consciente de sus propias áreas de conflicto para que su personalidad y problemas no interfieran con el trabajo con sus pacientes (Roque Tovar \& García García, 2016). Además, esta particularidad se podría explicar por la preocupación local acerca del estado de salud mental de los profesionales referida por Casari (2010).

En Argentina esto cobra un sentido, en especial, por la histórica lucha por la construcción del rol del psicólogo en general y del psicoterapeuta en particular (Caló, 2000; González \& Dagfal, 2012). Este trabajo, que trasciende los diferentes modelos de psicoterapias, se propone contribuir a la superación de las diferentes batallas por la defensa del campo de intervención, el esclarecimiento del campo de acción del profesional y el otorgamiento del estatus de ciencia con la concomitante proliferación de estudios científicos que abonen el campo de las psicoterapias.

Si bien se trata de un estudio de tipo exploratorio, y por lo tanto no se pudo contar con estudios previos, es muy relevante poder sistematizar el quehacer profesional, la formación $\mathrm{y}$ las competencias requeridas atravesando transversalmente los principales modelos teóricosprincipalmente en un país con tanta trayectoria en psicoterapias. A partir de estos resultados, se requeririan estudios adicionales, por un lado, para determinar el peso relativo de cada competencia, y por otro, el desarrollo de modelos propios de cada subespecialización de la psicoterapia y de las particularidades atenientes a cada modelo teórico, como ya se han realizado en otros países (Newman, 2010; Pilling, Roth, \& Stratton, 2010).

\section{REFERENCIAS}

American Psychological Association (2010). Ethical Principles of Psychologists and Code of Conduct. Recuperado de https://www.apa.org/ethics/code/

ANECA. (2005). Libro Blanco para El Titulo de Grado de Psicología. Recuperado de http://www.aneca.es/var/media/150356/libroblanco_psicologia_def.pdf

Ardila Suárez, E. E. \& Rueda Arenas, J. P. (2013). La saturación teórica en la teoría fundamentada: su delimitación en el análisis de trayectorias de vida de víctimas del desplazamiento forzado en Colombia. 
Revista Colombiana de Sociología 36(2) 93-114.

Bartram, D., Bamberg, E., Bräuner, B., Georgas, J., Holte, A., Jern, S., \& Job, R. (2008). EuroPsy - The European Certificate in Psychology. Recuperado de http://www.inpa-europsy.it/nuovi. docum.2008/EuroPsy_english.pdf

Benatuil, D., \& Laurito, M. J. (2017). Specific competences in the Tuning Latin America Project: their degree of importance and achievement among a sample of psychology students. Tuning Journal for Higher Education, 4(2), 333-351. https://doi.org/10.18543/tjhe-4(2)-2017pp333-351

Beneitone, P., Esquetine, C., González, J., Marty, M., Siufi, G., \& Wagennar, R. (2007). Reflexiones y perspectivas de la educación superior en América Latina: Informe final del proyecto Tuning América Latina 2004-2007. Bilbao: Universidad de Deusto.

Berdullas, M., Peiro, J., Ramos, J., Álvarez, M., Del Barrio, M., Urra, J., ... Puente, H. (2007). Competencias profesionales del psicólogo. Consejo General de Colegios Oficiales de Psicólogos EuroPsy. Recuperado de http:// www.europsy.cop.es /index.php?page=competencias-de-los-psicologos

Botella, L. \& Maestra, J. (2016). Integración en psicoterapia en terapeutas en formación: atribución de factores comunes e ingredientes específicos en casos prototípicos de cinco orientaciones psicoterapéuticas. Revista Argentina de Clinica Psicológica, 25(1), 39-48.

Boyatzis, R. E. (1998). Transforming qualitative information: Thematic analysis and code development. London: Sage Publications, Inc.

Braun, V. \& Clarke, V. (2006) Uso del análisis temático en psicología. Investigación cualitativa en psicología, 3, 77-101.

Caló, O. (2000). Ética y deontología en la formación del psicólogo argentino. Fundamentos en Humanidades, $1(2), 7-12$

Casari, L. (2010). ¿Es necesaria la salud mental en los psicólogos? Diálogos, 1(2), 29-41.

Colegio de Psicólogos de Tucumán. (2018). Incumbencias Profesionales de los Psicólogos. Recuperado de http://colpsicologostuc.org.ar/noticias/asuntos-profesionales/incumbencias-profesionales-de-lospsicologos/.

Corbin, J. \& Srauss, A. (1990). Grounded Theory Research: Procedures, Canons, and Evaluative Criteria. Qualitative Sociology, 13(1)3-21. https://doi.org/10.1007/BF00988593

Cranney, J., Turnbull, C., Provost, S. C., Martin, F., Katsikitis, M., Whithe, F.A., ... Varcin, K.J. (2009). Graduate attributes of the 4-year Australian undergraduate psychology program. Australian Psychologist, 44(4): 253-262. https://doi.org/10.1080/00050060903037268

Daniel, J. H., Roysircar, G., Abeles, N., \& Boyd, C. (2004). Individual and cultural-diversity competency: Focus on the therapist. Journal of Clinical Psychology, 60(7), 755-770. https://doi.org/10.1002/jclp.20014

Edwards, H. (2000). A framework for the determination of competencies in relation to mobility for psychology under AIT. Canadian psychological Association. Recuperado de https://www.cpa.ca/documents/Framework.PDF

European Association for Psychotherapy. (2017). The European Certificate of Psychotherapy. Recuperado de https://www.europsyche.org/download/cms/100510/ECP-document-version-7-0-voted-AGM-ViennaFeb-2017_offic.pdf

European Certificate in Psychology. (2011). EuroPsy - the European Certificate in Psychology. Recuperado de http://www.unideusto.org/tuningeu/images/stories/Summary_of_outcomes_TN/Psychology_referenc e_points.pdf

Fernández-Álvarez, H. (1994). Fundamentos de un modelo integrativo en psicoterapia. Buenos Aires: Paidós.

Fernández-Álvarez, H., Pérez, Al. \& Fraga Míguez, M. (2008). Modelo de abordaje y diseño de tratamientos En H. Fernández-Álvarez (Ed.), Integración y salud mental. El proyecto AIGLÉ 1977-2008 (pp.23-64). Bilbao: Desclée de Brouwer.

Fierro, C. \& Di Doménico, C. (2016). Pluralismo crítico: Historia de la ciencia en debates sobre formación en psicología. Quaderns de Psicologia, 18(2), 27-57. https://doi.org/10.5565/rev/qpsicologia.1322

Fuentes Pallas, M. J. \& Lorenzo Pontevedra, M. C. (2008). El Proceso Terapéutico. En L. A. Oblitas (Ed.), Psicoterapias contemporáneas (pp.1-14). Santa Fe: Cengage Learning.

García, F. S. \& Fernández-Álvarez, H. (2001). Investigación en psicoterapia: un puente entre teoría y clínica. VERTEX Revista Argentina de Psiquiatría, 12, 204-210.

González, M. E. \& Dagfal, A. (2012). El psicólogo como psicoanalista: problemas de formación y autorización. Entre la universidad y las instituciones. Revista Electrónica de la Facultad de Psicología de la Universidad de Buenos Aires Intersecciones Psi, 2(5) 12-18.

Gonsalvez, C. J., Hamid, G., Savage, N. M., \& Livni, D. (2017). The Supervision Evaluation and Supervisory Competence Scale: Psychometric Validation. Australian Psychologist, 52(2) 94-103. https://doi.org/10.1111/ap.12269

Herrera Rojas, A. (1993). La medición en psicología. Bogotá: Universidad de Bogotá.

International Association of Applied Psychology. (2016). Declaración Internacional de Competencias Fundamentales en Psicologia Profesional. Recuperado de http://observatorio.ascofapsi.org.co/static/documents/IPCPCompetenciasProfesionalesPsicologia.pdf

Kaslow, N., Rubin, N., Bebeau, M., Leigh, I., Lichtenberg, J., Nelson, P., ... Smith, I. (2007). Competency Assessment Models. Professional Psychology: Research and Practice 38(5), 463-473. https://doi.org/10.1037/0735-7028.38.5.463

Kenkel, M. B., \& Peterson, R. L. (2010). Competency-based education for professional psychology. American Psychological Association. https://doi.org/10.1037/12068-000

Klappenbach, H. (2015). La formación universitaria en psicologia en Argentina: perspectivas actuales y desafios a la luz de la historia. Universitas Psychologica, 14(3), 937-960. https://doi.org/10.11144/Javeriana.upsy14-3.fupa 
Klinar, D., Gago, P. \& Alonso, M. (2019). Distribución ocupacional de los/as psicólogos/as de la República Argentina. Relevamiento 2019. Póster presentado en el XI Congreso Internacional de Investigación y Práctica Profesional en Psicologia. XXVI Jornadas de Investigación. XV Encuentro de Investigadores en Psicología del MERCOSUR. I Encuentro de Investigación de Terapia Ocupacional. I Encuentro de Musicoterapia. Facultad de Psicología - Universidad de Buenos Aires, Buenos Aires.

Laurito, M. J. \& Benatuil, D. (2017). Distancia entre los niveles de importancia y logro de las competencias especificas en muestra de graduados de una carrera de psicologia. Revista de Psicología, 13(25), 71-83.

Laws, H. B., Constantino, M. J., Sayer, A. G., Klein, D. N., Kocsis, J. H., Manber, R., ... Arnow, B. A. (2017). Convergence in patient-therapist therapeutic alliance ratings and its relation to outcome in chronic depression treatment. Psychotherapy Research, 27(4), 410-424. https://doi.org/10.1080/10503307.2015.1114687

Martínez, F. M. \& Carmona, G. (2009). Aproximación al Concepto de "Competencias Emprendedoras": Valor Social e Implicaciones Educativas. Revista Iberoamericana sobre Calidad, Eficacia y Cambio en Educación, 7(3), 82-98.

Mieles Barrera, M., Tonon, G., \& Alvarado Salgado, S. (2012). Investigación cualitativa: el análisis temático para el tratamiento de la información desde el enfoque de la fenomenología social. Universitas Humanistica, 74, 195-225.

Ministerio de Educación y Justicia de la Nación (1985). Resolución N 2447/85. Recuperado de https://www.cppm.org.ar/wp-content/uploads/2012/04/incumbencias-al-titulo.pdf

Mutual Recognition Agreement. (2004). Mutual Recognition Agreement of the Regulatory Bodies for Professional Psychologists in Canada. Recuperado de https://cpa.ca/docs/File/MRA.pdf

New Zealand Psychologist Board. (2011). Core Competencies for the practice of psychology in New Zealand. Recuperado de http://www.psychologistsboard.org.nz/cms_show_download.php?id=41

Newman, C.F. (2010). Competency in conducting cognitive-behavioral therapy: foundational, functional, and supervisory aspects. Psychotherapy: Theory, Research, Practice, Training, 47(1) 12-19. https://doi.org/10.1037/a0018849

Pedrosa, I., Suárez-Álvarez, J., \& García-Cueto, E. (2013). Evidencias sobre la validez de contenido: avances teóricos y métodos para su estimación. Acción Psicológica, 10(2), 3-18. https://doi.org/10.5944/ap.10.2.11820

Pilling, S. Roth, A.D. \& Stratton, P. (2010). The competences required to deliver effective Systemic Therapies. Recuperado de http:/ /www.inpa-europsy.it/moduli/EuroPsy\%20Regulations\%20July\%202011.pdf

Rodolfa, E., Bent, R., Eisman, E., Nelson, P., Rehm, L., \& Rithcie, P. (2005). A Cube Model for Competency Development: Implications for Psychology Educators and Regulators. Professional Psychology: Research and Practice 36(4), 347-354. https://doi.org/10.1037/0735-7028.36.4.347

Roque Tovar, B., \& García García, L. (2016). Elaboración de la experiencia profesional: una propuesta de aprendizaje reflexivo en la formación del psicólogo. Enseñanza e Investigación en Psicología, 21(2), 113119.

Universidad de La Plata. (2018). Actividades Reservadas: Declaración Conjunta. Recuperado de http://www.psico.unlp.edu.ar/articulo/2018/5/22/actividades_reservadas_declaracion_conjunta

Urrutia Egaña, M., Barrios Araya, S., Gutiérrez Núñez, M., \& Mayorga Camus, M. (2014). Métodos óptimos para determinar validez de contenido. Educación Médica Superior, 28(3), 547-558.

Zilcha-Mano, S. (2017). Is the alliance really therapeutic? Revisiting this question in light of recent methodological advances. American Psychologist, 72(4), 311-325. https://doi.org/10.1037/a0040435

Recibido 13-05-2020 | Aceptado 18-08-2020

Este trabajo se encuentra bajo una Licencia Creative Commons Atribución 4.0 Internacional que permite a terceros utilizar lo publicado siempre que se dé el crédito pertinente a los autores y a Psicodebate 